\title{
Correction: Metabolic syndrome increases the risk of upgrading and upstaging in patients with prostate cancer on biopsy: a radical prostatectomy multicenter cohort study
}

\section{Cosimo De Nunzio ${ }^{1}$ - Aldo Brassetti ${ }^{1}$ - Giuseppe Simone $\mathbb{1}^{2} \cdot$ Riccardo Lombardo $^{1} \cdot$ Riccardo Mastroianni $^{2}$. Devis Collura ${ }^{1} \cdot$ Giovanni Muto $^{2,3} \cdot$ Michele Gallucci $^{1} \cdot$ Andrea Tubaro $^{1}$}

Published online: 1 February 2019

(c) Springer Nature America, Inc. 2019

Correction to: Prostate Cancer Prostatic Dis. 4, 108 (2018) https://doi.org/10.1038/s41391-018-0054-9 published online 4 June 2018.

The original version of this Article contained an error in the spelling of the authors Cosimo De Nunzio, Aldo Brassetti, Giuseppe Simone, Riccardo Lombardo, Riccardo
Mastroianni, Devis Collura, Giovanni Muto, Michele Gallucci and Andrew Tubaro, which were incorrectly given as De Nunzio Cosimo, Brassetti Aldo, Simone Giuseppe, Lombardo Riccardo, Mastroianni Riccardo, Collura Devis, Muto Giovanni, Gallucci Michele and Tubaro Andrea. This has now been corrected in both the PDF and HTML versions of the Article.

Cosimo De Nunzio

cosimodenunzio@virgilio.it

1 Department of Urology, Ospedale Sant'Andrea, "La Sapienza" University, Rome, Italy

2 Department of Urology, "Regina Elena" National Cancer Institute, Rome, Italy

3 Department of Urology, "San Giovanni Bosco" Hospital, Turin, Italy 